\title{
Bidimensional Spectroscopy with Optical Fibers of Spatially Resolved Emission-Line Regions Around Active Galactic Nuclei
}

\author{
S. Arribas, E. Mediavilla, C. del Burgo, and B. García-Lorenzo \\ Instituto de Astrofśica de Canarias, 38200-La Laguna, Tenerife, Spain
}

\begin{abstract}
We summarize our experience in observing the circumnuclear regions of AGN with the optical-fiber systems that we have developed for the William Herschel and Nordic Optical Telescopes at La Palma.
\end{abstract}

\section{Introduction}

When spectroscopy of extended regions (e.g., the spatially resolved emission-line regions) is required, the problem arises of how to record three variables $(\alpha, \delta, \lambda)$ into a two-dimensional detector. Traditionally, this has been solved by sequential procedures, which use time for scanning a third variable. In particular, the classical technique of long-slit scans consists in recording a set of 2-D images [e.g., $(\alpha, \lambda)$ ] changing one angular variable $(\delta)$ with time. Similarly, FabryPérot interferometers record a set of 2-D images $(\alpha, \delta)$, changing the wavelength coordinate $(\lambda)$ with time.

In recent years new techniques have been developed in order to pack three variables $(\alpha, \delta, \lambda)$ into the two spatial dimensions of the current detectors. These techniques, generically called 'integral field spectroscopy' (IFS), imply a geometrical transformation of the image at the telescope focal plane. This transformation can be done with (1) an array of microlenses (TIGER: Courtes et al. 1988), (2) optical fibers (SILFID/ARGUS: Vanderriest \& Lemonier 1988; HEXAFLEX: Arribas, Mediavilla, \& Rasilla 1991), (3) mirrors acting as image slicers (3D: Genzel, this volume), or (4) a combination of microlenses and optical fibers (MPFS: Afanasiev et al. 1990). Approach (1) can give full spatial coverage of the field, but is more restricted from a spectral point of view. Conversely, in approach (2) the geometry of the fibers does not permit a full coverage of the object, though it allows good spectral coverage and resolution. Perhaps the best solution from a conceptual point of view is (4) as it permits full spatial coverage, and imposes no serious restrictions from a spectral point of view. However, in practice, it is difficult to implement, especially for intermediate- and small-size telescopes on account of their reduced focal-plane scales.

The present communication is about our experience observing the circumnuclear regions of AGN with optical-fiber systems (approach 2) to perform 2D spectroscopy. 


\section{The Concept}

Bidimensional spectroscopy with optical fibers allows simultaneous spectroscopy of many regions of an extended object thanks to the use of optical-fiber bundles with the following basic layout: at one end of the bundle, the fibers form a twodimensional array, which is connected to the telescope focal plane. At the other end, the fibers are aligned and connected at the entrance of the spectrograph, simulating the slit (a 'pseudoslit'). Therefore, when the telescope points to an extended object, each fiber transmits the light coming from a region of the object. At the detector, we obtain simultaneously a set of spectra, each one corresponding to a particular fiber or region of the object. We can reconstruct $2 \mathrm{D}$ images of any spectral feature contained in the spectra in software (e.g., line-intensity maps, velocity fields, continua, etc).

\section{Advantages and Limitations}

This technique has two main advantages. First, all the spatial and spectral information is simultaneously recorded (this property is shared with the other IFS techniques). This not only implies a more efficient way of observing, but perhaps more important, all the information is recorded under the same atmospheric and instrumental conditions. Second, it allows observation over wide spectral ranges at good resolution. Therefore, many different lines and indices can be simultaneously observed in two dimensions.

There are other practical advantages, such as as: (1) the spectra are accurately located in sky coordinates, $(2)$ it is possible to reduce the contamination inside the spectrograph by separating the fibers at the slit (this is important when observing in the vicinity of the active nucleus), (3) there is no need for accurate centering of the object, (4) the differential atmospheric effects can be corrected at the reduction stage, which (5) are basically performed following standard and simple procedures.

A specific limitation of this technique, when compared with other IFS approaches, is that the geometry of the fibers does not allow full spatial coverage of the region to be observed. A limitation shared with the other IFS approaches is the relatively small spatial coverage (typically, $\sim 10^{\prime \prime} \times 10^{\prime \prime}$ ) when compared, for instance, to Fabry-Pérots. This is due to the limited number of spectra which can be located at the detector (or number of fibers at the slit). However, this is not so important when dealing with objects of relatively small angular size, such as the spatially resolved emission-line regions of AGN. Of course, when the object is larger than the field of view of the bundle, several exposures can be taken, at the cost of losing simultaneity.

\section{The Systems}

Among the various systems developed by other groups, we would like to mention SILFID/ARGUS (Vanderriest \& Lemonier 1988), Densepack (Barden \& Wade 1988), and the Multi-Pupil Fiber Spectrograph (Afanasiev et al. 1990).

Our group has built several experimental systems for the WHT and NOT. HEXAFLEX (Arribas et al. 1991), which was equipped with a 61-fiber bundle, 
each fiber $0^{\prime \prime} .9$ in diameter was first installed at the WHT in 1989. This system is no longer operating at the WHT. It was fully redesigned to adapt to the NOT, and now known as HEXAFLEX-II; García et al. 1994). We also changed the optical-fiber bundle, which now has 125 fibers $0^{\prime \prime .} .7$ in diameter. For the WHT, we built a new system, called 2D-FIS (two-dimensional Fiber ISIS system) and it is described by García et al. (1994). The bundle has 125 fibers $0^{\prime \prime} .9$ in diameter. This system combines the advantages of the optical-fiber systems with the use of the ISIS double spectrograph (Carter et al. 1993).

We are now building, in collaboration with the RGO, a new system for the WHT, called INTEGRAL. It will use a specially designed spectrograph for working with fibers (WYFFOS) built by the RGO. It will have five bundles, with fibers from $0^{\prime \prime} .45$ to $3^{\prime \prime}$ in diameter.

It is interesting to mention the instruments for the VLT-3 (FUEGOS; Félenbock et al. 1994) and GEMINI (IFU: Allington-Smith et al. 1994) telescopes. There are also projects in progress for building IFS instruments based on the use of optical fibers and microlenses (approach 4).

\section{Galaxies Studied}

Thanks to the systems for the WHT and NOT telescopes mentioned above, we are developing a program for the study of the circumnuclear regions of active galactic nuclei. The following galaxies have been analyzed so far:

NGC 4151: Mediavilla et al. (1992) observed this galaxy with HEXAFLEX, obtaining the velocity field of the ionized gas, and the ionization structure in the central $13^{\prime \prime}$ from the $\mathrm{H} \alpha-[\mathrm{NII}]-[\mathrm{SII}]$ spectral region. Observations with HEXAFLEX-II in the $\mathrm{H} \beta-[\mathrm{O} \mathrm{III}]$ region (Mediavilla \& Arribas 1995), confirm that the velocity field has two receding poles, and establish the identification of the optical nucleus with the galaxy's mass centroid.

NGC 5728: HEXAFLEX observations of this galaxy (Arribas \& Mediavilla 1993) suggested that its nucleus was obscured, with location at the center of rotation of the ionized gas. This location turned out to be at the apex of a well-defined biconical structure in the HST images by Wilson et al. (1993). Further observations of this galaxy with HEXAFLEX-II in the $\mathrm{H} \beta-[\mathrm{O}$ III] region (Mediavilla \& Arribas, 1995) confirmed these results, supporting the idea that this galaxy is an archetypal Seyfert 2 galaxy, in the framework of the standard AGN models.

NGC 3227: HEXAFLEX and 2D-FIS observations (Mediavilla \& Arribas 1993; Arribas \& Mediavilla 1994) revealed that the nucleus (BLR) of this Seyfert 1 galaxy is off-set with respect to the center of rotation of the ionized gas, and with respect to the maximum of the gas velocity dispersion.

NGC 1068: HEXAFLEX-II observations (Arribas et al. 1996) allowed a kinematical decomposition of the different gaseous components in the inner region 
of this galaxy to be performed. A study of the intensity distribution and the velocity field of each component led to the identification of a biconical (or bipolar) structure in the inner region of NGC 1068. The center/apex of this structure, which is coincident with the kinematic center of the ionized gas, was suggested to be the location of the hidden nucleus (see also García-Lorenzo et al., this volume). Other galaxies, such as NGC 7331 and NGC 3516, are now under study.

Other groups are also developing similar programs on the basis of IFS data (e.g., Pecontal, Ferruit, \& Wilson 1995; Ferruit \& Pecontal, this volume; Afanasiev et al. 1995, and references therein).

\section{References}

Afanasiev, V.I., et al. 1990, Acad. Nauk. USSR, preprint 54.

Afanasiev, V.I., et al. 1995, ASP Conf. Ser. Vol. 71, 269.

Allington-Smith, J., \& Zhang, J., 1995, in Scientific and Engineering Frontiers for 8-10 m. Telescopes, Tokyo.

Arribas, S., \& Mediavilla, E. 1993, ApJ, 410, 149.

Arribas, S., \& Mediavilla, E. 1994, ApJ, 437, 552.

Arribas, S., Mediavilla, E., \& Rasilla, J.L., 1991, ApJ, 369, 260.

Arribas, S., Mediavilla, E., \& García-Lorenzo, B. 1996, ApJ, 463, 509.

Barden, S., \& Wade, R. 1988, ASP Conf. Ser. Vol. 3, 113.

Carter, D., et al. 1993, Isaac Newton Group, La Palma. User Manual No. XXIV.

Courtes, G., et al. 1988, in Instrumentation for Ground-Based Astronomy, ed. L. B. Robinson, Springer-Verlag, p. 267.

Félenbock, P. et al., 1994, in Instrumentation in Astronomy, Vol 2198, ed. D. L. Crawford \& E. R. Craine (Hawaii: SPIE Symp.), 115.

Ferruit, P.,\& Pecontal, E., 1997, this volume.

García, A., et al. 1994, in Instrumentation in Astronomy, Vol 2198, ed. D. L. Crawford \& E..R. Craine (Hawaii: SPIE Symp.), 75.

García-Lorenzo, B., Arribas, S., \& Mediavilla, E., 1997, this volume.

Genzel, R., 1997, this volume.

Mediavilla, E., Arribas, S., \& Rasilla, J.L. 1992, ApJ, 396, 517.

Mediavilla, E., \& Arribas, S. 1993, Nature, 365, 420.

Mediavilla, E., \& Arribas, S. 1995, MNRAS, 276, 579.

Pecontal, E., Ferruit, P., Wilson, A.S. 1995, ASP Conf. Ser. Vol. 71, 269.

Vanderriest, C., \& Lemonnier. J. P. 1988, in 'Instrumentation for Ground-Based Astronomy', ed. L. B. Robinson, Springer-Verlag, p. 304.

Wilson, A.S., et al. 1993, ApJ, 419, L61. 